\title{
SOME SUBMERSIONS OF CR-HYPERSURFACES OF KAEHLER-EINSTEIN MANIFOLD
}

\author{
VITTORIO MANGIONE
}

Received 26 April 2002

\begin{abstract}
The Riemannian submersions of a CR-hypersurface $M$ of a Kaehler-Einstein manifold $\tilde{M}$ are studied. If $M$ is an extrinsic CR-hypersurface of $\tilde{M}$, then it is shown that the base space of the submersion is also a Kaehler-Einstein manifold.
\end{abstract}

2000 Mathematics Subject Classification: 53B35, 53C25.

1. Introduction. The study of the Riemannian submersions $\pi: M \rightarrow B$ was initiated by O'Neill [14] and Gray [9]. This theory was very much developed in the last thirty five years. Besse's book [3, Chapter 9] is a reference work. Bejancu introduced a remarkable class of submanifolds of a Kaehler manifold that are known as CR-submanifolds (see [1, 2]). On a CR-submanifold, there are two complementary distributions $D$ and $D^{\perp}$, such that $D$ is $J$-invariant and $D^{\perp}$ is $J$-anti-invariant with respect to the complex structure $J$ of the Kaehler manifold. The integrability of the anti-invariant distribution $D$ was proved by Blair and Chen [4].

Recently, Kobayashi [10] considered the similarity between the total space of a Riemannian submersion and a CR-submanifold of a Kaehler manifold in terms of the distribution. He studied the case of a generic CR-submanifolds in a Kaehler manifold and proved that the base space is a Kaehler manifold.

In Section 3, we extend the result of Kobayashi to the general case of a CRsubmanifold.

In Section 4, we study a Riemannian submersion from an extrinsic hypersurface $M$ of a Kaehler-Einstein manifold $\tilde{M}$ onto an almost-Hermitian manifold $B$. In this case, we prove that the basic manifold is a Kaehler-Einstein manifold. If $\tilde{M}$ is $C^{n+1}$, a standard example is the Hopf fibration $S^{2 n+1} \rightarrow C P^{n}$ equipped with the canonical metrics.

For the basic formulas of Riemannian geometry, we use [11, 12].

2. Preliminaries. Let $\tilde{M}$ be a complex $m$-dimensional Kaehler manifold with complex structure $J$ and Hermitian metric $\langle\cdot, \cdot\rangle$. Bejancu [2] introduced the concept of a CR-submanifold of $\tilde{M}$ as follows: a real Riemannian manifold $M$, isometrically immersed in a Kaehler manifold $\tilde{M}$, is called a CR-submanifold of $\tilde{M}$ if there exists on $M$ a differentiable holomorphic distribution $D$ and its 
orthogonal complement $D^{\perp}$ on $M$ is a totally real distribution, that is, $J D_{x}^{\perp} \subseteq$ $T_{x}^{\perp} M$, where $T_{x}^{\perp} M$ is the normal space to $M$ at $x \in M$ for any $x \in M$. It is easily seen that each real orientable hypersurface of $M$ is a CR-submanifold. The Riemannian metric induced on $M$ will be denoted by the same symbol $\langle\cdot, \cdot\rangle$.

Let $\tilde{\nabla}$ (resp., $\nabla$ ) be the operator of covariant differentiation with respect to the Levi-Civita connection on $\tilde{M}$ (resp., $M$ ). The second-fundamental form $B$ is given by

$$
B(E, F)=\tilde{\nabla}_{E} F-\nabla_{E} F
$$

for all $E, F \in \Gamma(T M)$, where $\Gamma(T M)$ is the space of differentiable vector field on $M$. We denote everywhere by $\Gamma(\tau)$ the space of differentiable sections of a vector bundle $\tau$.

For a normal vector field $N$, that is, $N \in \Gamma\left(T^{\perp} M\right)$, we write

$$
\tilde{\nabla}_{E} N=-L_{N} E+\nabla_{E}^{\perp} N
$$

where $-L_{N} E$ (resp., $\nabla_{E}^{\frac{1}{E}} N$ ) denotes the tangential (resp., normal) component of $\tilde{\nabla}_{E} N$.

Let $\mu$ be the orthogonal complementary vector bundle of $J\left(D^{\perp}\right)$ in $T^{\perp} M$, that is, $T^{\perp} M=J\left(D^{\perp}\right) \oplus \mu$.

It is clear that $\mu$ is a holomorphic subbundle of $T^{\perp} M$, that is, $J \mu=\mu$.

DEFINITION 2.1 (Kobayashi [10]). Let $M$ be a CR-submanifold of a Kaehler manifold $\tilde{M}$. A submersion from a CR-manifold $M$ onto an almost-Hermitian manifold is a Riemannian submersion $\pi: M \rightarrow M^{\prime}$ with the following conditions:

(i) $D^{\perp}$ is the kernel of $\pi_{*}$,

(ii) $\pi_{*}: D_{x} \rightarrow T_{\pi(x)} M^{\prime}$ is a complex isometry for every $x \in M$.

This definition is given by Kobayashi for the case where $\mu$ is a null subbundle of $T^{\perp} M$ (see [10]). If $J D_{x}^{\perp}=T_{x}^{\perp} M$ for any $x \in M$, we say that $M$ is a generic CR-submanifold of $\tilde{M}$ (Yano and Kon [15]). For example, any real orientable hypersurface of $\tilde{M}$ is a generic CR-submanifold of $\tilde{M}$.

Concerning the basic notions on the Riemannian submersions, see O'Neill [14] and Gray [9].

The vertical distribution of a Riemannian submersion is an integrable distribution. In our case, the distribution vertical is $D^{\perp}$, which is integrable according to a theorem by Blair and Chen [4].

The sections of $D^{\perp}$ (resp., $D$ ) are called the vertical vector fields (resp., the horizontal vector fields) of the Riemannian submersion $\pi: M \rightarrow M^{\prime}$. The letters $U, V, W$, and $W^{\prime}$ will always denote vertical vector fields, and the letters $X, Y$, $Z$, and $Z^{\prime}$ denote horizontal vector fields. For any $E \in \mathscr{L}(M), v E$ and $h E$ denote the vertical and horizontal components of $E$, respectively. A horizontal vector field $X$ on $M$ is said to be basic if $X$ is $\pi$-related to a vector field $X^{\prime}$ on $M^{\prime}$. 
It is easy to see that every vector field $X^{\prime}$ on $M^{\prime}$ has a unique horizontal lift $X$ to $M$, and $X$ is basic.

Conversely, let $X$ be a horizontal vector field and suppose that $\langle X, Y\rangle_{X}=$ $\langle X, Y\rangle_{y}$ for all $Y$ basic vector fields on $M$, for all $x, y \in \pi^{-1}\left(x^{\prime}\right)$, and for all $x^{\prime} \in M^{\prime}$. Then, the vector field $X$ is basic. We have the following O'Neill's lemma (see $[8,14])$.

LEMMA 2.2. Let $X$ and $Y$ be basic vector fields on $M$. Then, they are satisfying the following:

(i) the horizontal component $h[X, Y]$ of $[X, Y]$ is a basic vector field and $\pi_{*} h[X, Y]=\left[X^{\prime}, Y^{\prime}\right] \circ \pi$,

(ii) $h\left(\nabla_{X} Y\right)$ is a basic vector field corresponding to $\nabla_{X^{\prime}}^{\prime} Y^{\prime}$, where $\nabla^{\prime}$ is the Levi-Civita connection on $\left(M^{\prime},\langle,\rangle^{\prime}\right)$,

(iii) $[X, U] \in \Gamma\left(D^{\perp}\right)$ for any vertical field $U \in \Gamma\left(D^{\perp}\right)$.

We recall that a Riemannian submersion $\pi:(M, g) \rightarrow\left(M^{\prime}, g^{\prime}\right)$ determines the fundamental tensor field $T$ and $A$ by the formulas

$$
\begin{aligned}
& T_{E} F=h \nabla_{v E} v F+v \nabla_{v E} h F, \\
& A_{E} F=v \nabla_{h E} h F+h \nabla_{h E} v F,
\end{aligned}
$$

for all $E, F \in \Gamma(T M)$ (cf. O’Neill [14] and Besse [3]).

It is easy to prove that $T$ and $A$ satisfy

$$
\begin{aligned}
& T_{U} V=T_{v} U, \\
& A_{X} Y=\frac{1}{2} v[X, Y],
\end{aligned}
$$

for any $U, V \in \Gamma\left(D^{\perp}\right)$ and $X, Y \in \Gamma(D)$.

Formula (2.4) means that the restriction of $T$ to the integrable distribution $D^{\perp}$ is the second-fundamental form of the fiber submanifolds in $M$, and (2.5) measures the integrability of the distribution $D$.

We have the following properties:

$$
\begin{aligned}
& \nabla_{U} X=T_{U} X+h \nabla_{U} X, \\
& \nabla_{X} U=v \nabla_{X} U+A_{X} U, \\
& \nabla_{X} Y=h \nabla_{X} Y+A_{X} Y,
\end{aligned}
$$

for any $X, Y \in \Gamma(\mathscr{H})$ and $U \in \Gamma(\mathscr{V})$.

3. Kaehler structure on the basic space $M^{\prime}$. From (2.1), we have

$$
\tilde{\nabla}_{X} Y=h \nabla_{X} Y+v \nabla_{X} Y+\bar{h} B(X, Y)+\bar{v} B(X, Y)
$$

for any $X, Y \in \Gamma(D)$. 
Here, we denote by $h$ and $v$ (resp., $\bar{h}$ and $\bar{v}$ ) the canonical projections on $D$ and $D^{\perp}$ (resp., $\mu$ and $J D^{\perp}$ ). Define a tensor field $C$ on $M$ as the vertical component $v\left(\nabla_{X} Y\right)$ of $\nabla_{X} Y$ (cf. Kobayashi [10]). The tensor field $C$ is known to be a skew-symmetric tensor field defined by Kobayashi such that

$$
C(X, Y)=\frac{1}{2} v[X, Y]
$$

for all $X, Y \in \Gamma(D)$.

Note that the tensor field $C$ is the restriction of $A$ to $\Gamma(\mathscr{H}) \times \Gamma(\mathscr{H})$.

From Definition 2.1 and Lemma 2.2, we obtain that $J h \nabla_{X} Y$ (resp., $h \nabla_{X} J Y$ ) is a basic vector field and corresponds to $J^{\prime} \nabla_{X^{\prime}}^{\prime} Y^{\prime}$ (resp., $\nabla_{X^{\prime}}^{\prime} J^{\prime} Y^{\prime}$ ) for any basic vector fields $X$ and $Y$ on $M$.

On the Kaehler manifold $\tilde{M}$, we have

$$
\tilde{\nabla}_{E} J F=J \tilde{\nabla}_{E} F
$$

From (3.1) and (3.3), we obtain the following proposition.

Proposition 3.1. For any basic vector fields $X$ and $Y$ on $M$,

$$
\begin{aligned}
J h \nabla_{X} Y & =h \nabla_{X} J Y, \\
J C(X, Y) & =\bar{v} B(X, J Y), \\
C(X, J Y) & =J \bar{v} B(X, Y), \\
J \bar{h} B(X, Y) & =\bar{h} B(X, J Y) .
\end{aligned}
$$

THEOREM 3.2. Let $M$ be a CR-submanifold of a Kaehler manifold $\tilde{M}$ and $\pi: M \rightarrow M^{\prime}$ be a CR-submersion of $M$ on an almost-Hermitian manifold $M^{\prime}$. Then, $M^{\prime}$ is a Kaehler manifold.

Proof. From Lemma 2.2 and (3.4), we obtain that $\nabla_{X^{\prime}}^{\prime} J^{\prime} Y^{\prime}=J^{\prime} \nabla_{X^{\prime}}^{\prime} Y^{\prime}$, so that $M^{\prime}$ is a Kaehler manifold.

REMARK 3.3. Proposition 3.1 is proved for generic CR-submanifolds of $\tilde{M}$ (i.e., $\mu=0)$ in [10].

4. Riemannian submersions from extrinsic hyperspheres of EinsteinKaehler manifolds. We recall that a totally umbilical submanifold $M$ of a Riemannian manifold $\tilde{M}$ is a submanifold whose first-fundamental form and second-fundamental form are proportional.

The extrinsic hyperspheres are defined to be totally umbilical hypersurfaces, having nonzero parallel mean-curvature vector field (cf. Nomizu and Yano [13]). Many of the basic results concerning extrinsic spheres in Riemannian and Kaehlerian geometry were obtained by Chen [5, 6, 7]. 
Let $M$ be an orientable hypersurface in a Kaehler manifold $\tilde{M}$. Then, $M$ is an extrinsic hypersphere of $\tilde{M}$ if it satisfies

$$
B(E, F)=\langle E, F\rangle H
$$

for any vector fields $E$ and $F$ on $M$. Here, $H$ denote the mean-curvature vector field of $M$. If we put $k=\|H\|$ (where the norm $\|\cdot\|$ is, with respect to a scalar product, induced on every tangent space to $M$ ), then $k$ is a nonzero constant function on the extrinsic hypersphere $M$.

We denote by $N$ the global unit normal vector field to $M$. Then, $\xi=-J N$ is a global unit vector on $M$ such that $N=J \xi$. Let $D$ be the maximal $J$-invariant subspace (with respect to $J$ ) of the tangent space $T_{p} M$ for every $p \in M$. We see that $M$ is a CR-hypersurface of $M$ such that $T M=D \oplus D^{\perp}$, where $D^{\perp}$ is the one-dimensional anti-invariant distribution generated by the vector field $\xi$ on $M$.

The anti-invariant distribution $D^{\perp}$ is integrable, and its leaves are totally geodesic in $M$ (but not in $\tilde{M}$ ).

This is an easy consequence from Gauss and Weingarten's formulas of the leaves of $D^{\perp}$ in $M$. This means that O'Neill's tensor $T$ vanishes on the fibres of the Riemannian submersion $\pi: M \rightarrow B$.

The main result of this section is the following theorem.

THEOREM 4.1. Let $M$ be an orientable extrinsic hypersphere of an KaehlerEinstein manifold $\tilde{M}$. If $\pi: M \rightarrow B$ is a CR-submersion of $M$ on an almostHermitian manifold $B$, then $B$ is an Kaehler-Einstein manifold.

To prove Theorem 4.1, we need several lemmas.

LEMMA 4.2. Following the assumptions of Theorem 4.1, then

$$
\left\langle A_{x} \xi, A_{y} \xi\right\rangle=k^{2}\langle X, Y\rangle
$$

for any horizontal vector $X$ on $M$.

Proof. From Gauss's formula (2.1) and the umbilicality of $M$, we get $\tilde{\nabla}_{X} \xi=$ $\nabla_{X} \xi$ for any vector field $X$ on $M$. Then, we have

$$
\left\langle\tilde{\nabla}_{X} J N, Y\right\rangle=\left\langle\nabla_{X} \xi, Y\right\rangle=\left\langle h \nabla_{X} \xi, Y\right\rangle=\left\langle A_{X} \xi, Y\right\rangle
$$

On the other hand, $\tilde{M}$ is a Kaehler manifold, so that $\nabla$ commute with $J$ :

$$
\begin{aligned}
\left\langle\tilde{\nabla}_{X} J N, Y\right\rangle & =\left\langle J \tilde{\nabla}_{X} N, Y\right\rangle=-\left\langle\tilde{\nabla}_{X} N, J Y\right\rangle=\langle B(X, J Y), N\rangle \\
& =\langle G(X, J Y) H, N\rangle=k\langle X, J Y\rangle .
\end{aligned}
$$


Consequently,

$$
\left\langle A_{X} \xi, A_{Y} \xi\right\rangle=k\left\langle X, J A_{Y} \xi\right\rangle=-k\left\langle J X, A_{Y} \xi\right\rangle=k^{2}\langle X, Y\rangle .
$$

LEMMA 4.3. Following the assumptions of Theorem 4.1, then

$$
\left\langle A_{X} Y, A_{Z} W\right\rangle=k^{2}\langle X, J Y\rangle\langle Z, J W\rangle
$$

for any horizontal vector fields on $M$.

Proof. We say that $A_{X} Y$ is a vertical vector field, hence

$$
A_{X} Y=\left\langle A_{X} Y, \xi\right\rangle \xi
$$

Then,

$$
\left\langle A_{X} Y, A_{Z} W\right\rangle=\left\langle A_{X} Y, \xi\right\rangle\left\langle A_{Z} W, \xi\right\rangle=k^{2}\langle X, J Y\rangle\langle Z, J W\rangle
$$

LEMMA 4.4. Following the assumptions of Theorem 4.1, then

$$
\tilde{R}(X, Y, Z, W)=R(X, Y, Z, W)+k^{2}\{\langle X, Z\rangle\langle Y, W\rangle-\langle X, W\rangle\langle Y, Z\rangle\},
$$

where $\tilde{R}$ and $R$ are the curvature tensor on $\tilde{M}$ and $M$, respectively.

Proof. We have the Gauss equation

$$
\begin{aligned}
\tilde{R}(X, Y, Z, W)= & R(X, Y, Z, W)+\langle B(X, Z), B(Y, W)\rangle \\
& -\langle B(Y, Z), B(X, W)\rangle .
\end{aligned}
$$

Using the umbilicality condition, we get (4.9).

LEMMA 4.5. For any horizontal vector fields $X$ and $Y$ on $M$,

$$
\tilde{R}(\xi, X, Y, \xi)=0, \quad \tilde{R}(\xi, J X, J Y, \xi)=0 .
$$

PROof. For a Riemannian submersion with totally geodesic fibres, the following formula is known:

$$
\tilde{R}(X, V, Y, U)=\left\langle\left(\nabla_{V} A\right)(X, Y), U\right\rangle+\left\langle A_{X} V, A_{Y} U\right\rangle .
$$

On the other hand, the first term on the right part is skew-symmetric with respect to the vertical vector fields $V$ and $U$. From (4.12) and (4.9), we obtain (4.11). 
Proof of TheOrem 4.1. For the horizontal vector fields $X, Y, Z$, and $W$ on $M$, we have the following equation of O'Neill:

$$
\begin{aligned}
R(X, Y, Z, W)= & R^{\prime}\left(X^{\prime}, Y^{\prime} \cdot Z^{\prime}, W^{\prime}\right)-2\left\langle A_{X} Y, A_{Z} W\right\rangle \\
& +\left\langle A_{Y} Z, A_{X} W\right\rangle-\left\langle A_{X} Z, A_{Y} W\right\rangle
\end{aligned}
$$

(see $[3,14])$.

By (4.9) and (4.11), we get the following formula that connects the curvature of $M^{\prime}$ to the curvature of the Kaehler manifold $\tilde{M}$ :

$$
\begin{aligned}
\tilde{R}(X, Y, Z, W)= & R^{\prime}\left(X^{\prime}, Y^{\prime}, Z^{\prime}, W^{\prime}\right) \\
& -k^{2}\{\langle X, J Z\rangle\langle Y, J W\rangle-\langle X, J W\rangle\langle Y, J Z\rangle \\
& \quad+2\langle X, J Y\rangle\langle Z, J W\rangle\} \\
& -k^{2}\{\langle X, Z\rangle\langle Y, W\rangle-\langle X, W\rangle\langle Y, Z\rangle\} .
\end{aligned}
$$

Let $\left(e_{1}, \ldots, e_{p} ; J e_{1}, \ldots, J l_{p}\right)$ be a local $J$-frame of basic vector fields for the horizontal distribution $D$. Then, $\left(e_{1}, \ldots, e_{p}^{\prime} ; J^{\prime} e_{1}, \ldots, J^{\prime} e_{p}\right)$ is a local $J^{\prime}$-frame if $\pi_{\text {star }} e_{i}=e_{i}^{\prime}$ on the Kaehler manifold $B$.

Using the above lemmas, from (4.14) by a straightforward calculation, we conclude that $B$ is a Kaehler-Einstein manifold if $\tilde{M}$ is a Kaehler-Einstein manifold.

COROLLARY 4.6. Let $\tilde{M}$ be a complex-form space and $M$ an orientable $C R$ hypersurface of $\tilde{M}$. Then, the base space of submersion $\pi: M \rightarrow B$ is also a complex-form space.

PROOF. The corollary follows by straightforward calculation making use of (4.14).

EXAMPLE 4.7. Let $S^{2 n+1}$ be the standard hypersphere in $C^{n+1}$. Then, $S^{2 n+1}$ is an extrinsic hypersphere in $C^{n+1}$, and we have the Hopf fibration $\pi: S^{2 n+1} \rightarrow$ $C P^{n}$ equipped with the canonical metrics.

\section{REFERENCES}

[1] A. Bejancu, Real hypersurfaces of a complex projective space, Rend. Mat. (6) 12 (1979), no. 3-4, 439-445.

[2] _ Geometry of CR-Submanifolds, Mathematics and its Applications, vol. 23, D. Reidel, Dordrecht, 1986.

[3] A. Besse, Einstein Manifolds, Springer-Verlag, Berlin, 1984.

[4] D. E. Blair and B.-Y. Chen, On CR-submanifolds of Hermitian manifolds, Israel J. Math. 34 (1979), no. 4, 353-363.

[5] B.-y. Chen, CR-submanifolds of a Kaehler manifold. I, J. Differential Geom. 16 (1981), no. 2, 305-322.

[6] _ CR-submanifolds of a Kaehler manifold. II, J. Differential Geom. 16 (1981), no. 3, 493-509. 
[7] Geometry of Submanifolds and Its Applications, Science University of Tokyo, Tokyo, 1981.

[8] R. H. Escobales Jr., Riemannian submersions with totally geodesic fibers, J. Differential Geom. 10 (1975), 253-276.

[9] A. Gray, Pseudo-Riemannian almost product manifolds and submersions, J. Math. Mech. 16 (1967), 715-737.

[10] S. Kobayashi, Submersions of CR submanifolds, Tôhoku Math. J. (2) 39 (1987), no. $1,95-100$.

[11] S. Kobayashi and K. Nomizu, Foundations of Differential Geometry. Vol I, John Wiley \& Sons, New York, 1963.

[12] _ Foundations of Differential Geometry. Vol. II, Interscience Tracts in Pure and Applied Mathematics, no. 15, John Wiley \& Sons, New York, 1969.

[13] K. Nomizu and K. Yano, On circles and spheres in Riemannian geometry, Math. Ann. 210 (1974), 163-170.

[14] B. O'Neill, The fundamental equations of a submersion, Michigan Math. J. 13 (1966), 459-469.

[15] K. Yano and M. Kon, Structures on Manifolds, Series in Pure Mathematics, vol. 3, World Scientific, Singapore, 1984.

Vittorio Mangione: Dipartimento di Matematica, Universitá Degli Studi di Parma, Via Massimo D’Azeglio, 85/A, 43100 Parma, Italy

E-mail address: vittorio.mangione@unipr.it 


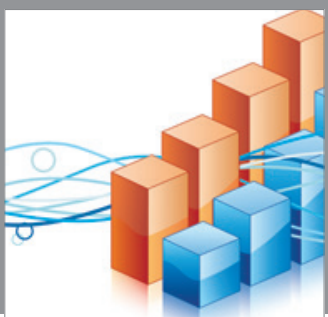

Advances in

Operations Research

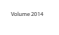

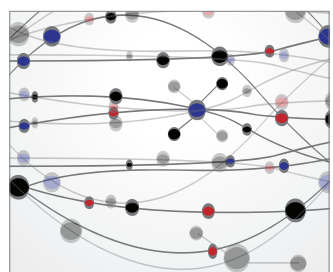

\section{The Scientific} World Journal
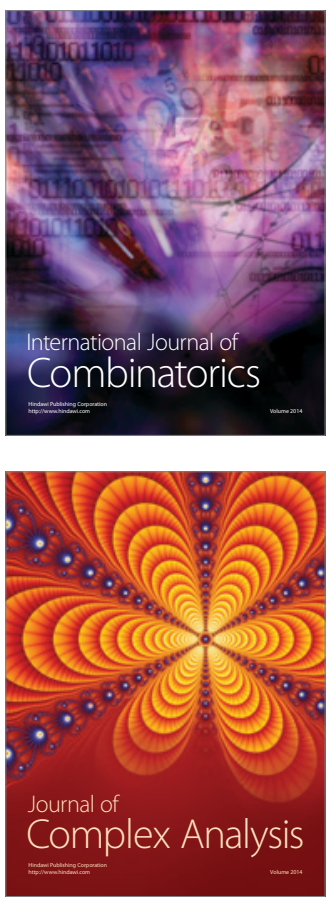

International Journal of

Mathematics and

Mathematical

Sciences
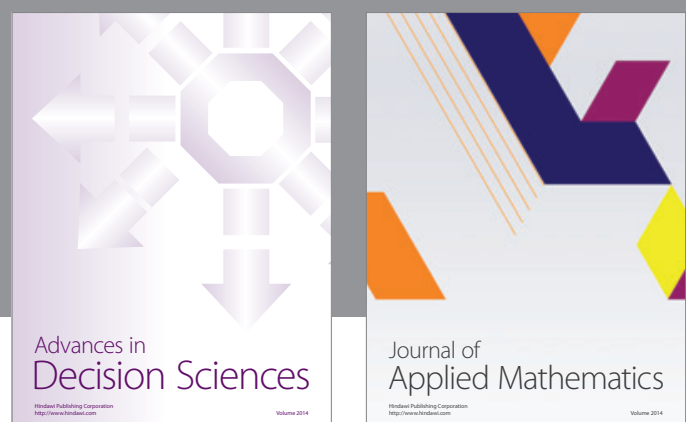

Journal of

Applied Mathematics
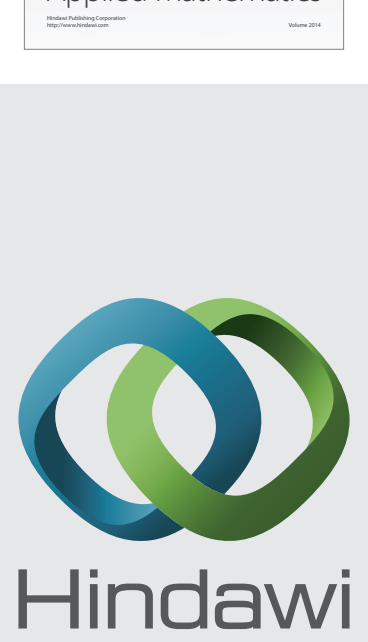

Submit your manuscripts at http://www.hindawi.com
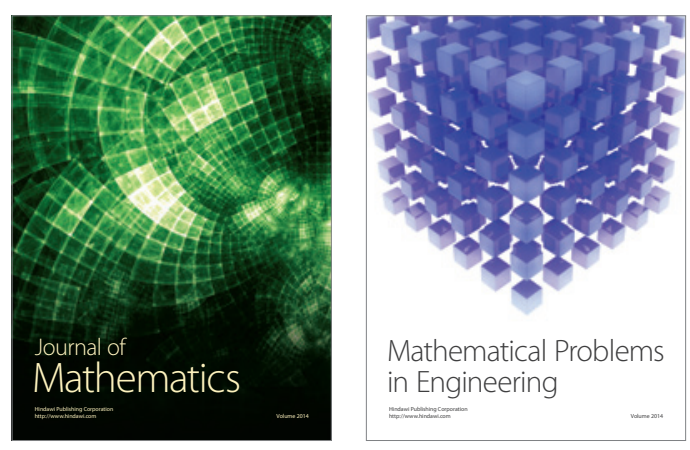

Mathematical Problems in Engineering
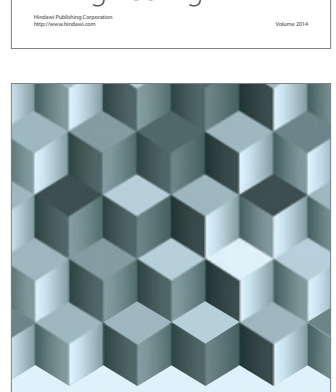

Journal of

Function Spaces
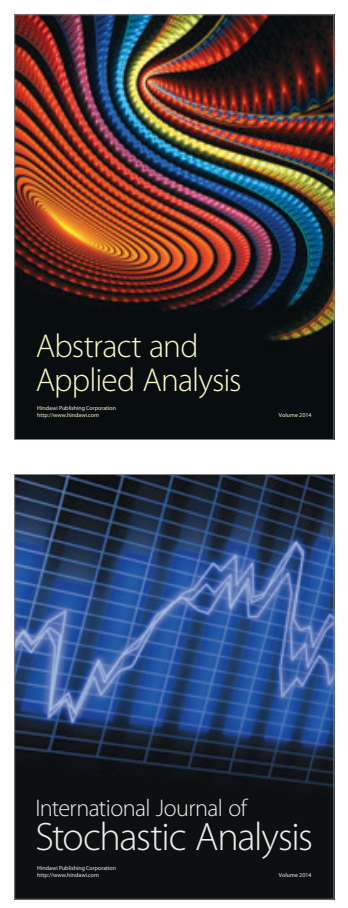

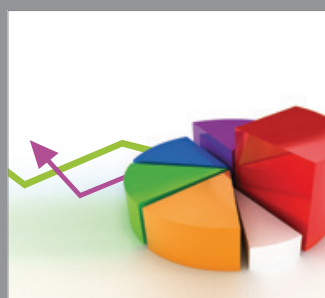

ournal of

Probability and Statistics

Promensencen
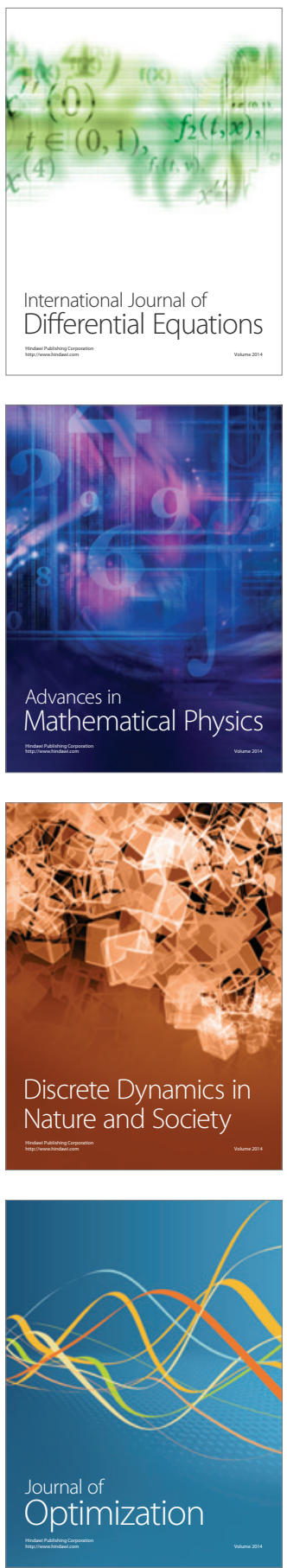